\title{
Avaliação da satisfação dos usuários com os serviços do consórcio intermunicipal de saúde do noroeste do Paraná
}

\author{
Evaluation of users satisfaction with the services of the \\ intermunicipal health consortia at the northwest of Paraná state
}

Erildo Vicente M uller ${ }^{1}$

$M$ arisabel Greco ${ }^{2}$

\footnotetext{
${ }^{1}$ Departamento de Enfermagem eSaúde Pública, Universidade Estadual de Ponta Grossa. Av. Gal. Carlos Cavalcanti 4.748/Bloco M, Campus U varanas. 84030-900 Ponta Grossa PR. erildomuller@uepg.br ${ }^{2}$ Curso deFarmáciae Bioquímica, Universidade Paranaense.
}

Abstract The health consortia between cities are made by small towns, this way they can have a bigger resolution in the attendancewith lower cost. In order to evaluate the satisfaction of the users with the services provided by the CISA of northwest Parana State, these users were interviewed through a standardized questionnaire. From a total of the 100 users $59 \%$ were women and $41 \%$ men. $27 \%$ of the users had basic education and $22 \%$ were illiterates. Regarding monthly income 58\% receive from 01 to 03 minimum wages and 42\% receive less than 01 minimum wage, $55 \%$ had already been consulted at CISA more than 6 times, and $44 \%$ answered that the city take less than a month to set appointments and $46 \%$ mentioned that had to wait 01 to 03 months to get an appointment. $57 \%$ related that the time taken by the city took to set the appointment were good, $43 \%$ thought that the time spent at consultation was also good. Regarding the professional that treated them, 68\% answered that it was a good professional and $56 \%$ said that the facilities of the CISA were great. It was concluded that the CISA is a reference to small towns, and that the users are satisfied with the services provided by them. Key words Satisfaction, U sers, Consortia, Health
Resumo Os consórcios intermunicipais de saúde são constituídos por municípios de pequeno porte, fazendo com que os mesmos tenham uma maior resolutividade no atendimento com menor custo. A fim de avaliar a satifisação dos usuários com os serviços prestados pelo CISA do noroeste do Paraná, foi realizada entrevista com os usuários do serviço saúde, através de um questionário padronizado. Dos 100 usuários, 59\% eram mulheres e $41 \%$, homens. $27 \%$ dos usuários possuem ensino fundamental ( 1 a a a 4 à séries) e $22 \%$ são analfabetos. $58 \%$ recebiam de um a três salários e $42 \%$, menos de um salário, $55 \%$ já consultaram no CISA mais de seis vezes, $44 \%$ responderam que o município leva menos de um mês para marcar a consulta e $46 \%$ esperam de um a três meses para conseguir uma consulta. Deacordo com o grau de satisfação, $57 \%$ relataram que o tempo que o município demorou para marcar a consulta foi bom, 43\% acham bom o tempo gasto durante a consulta, em relação ao profissional que os atendeu, $68 \%$ responderam ser um bom profissional e sobre as instalações do CISA, 56\% relataram quesão ótimas. Concluiu-se que o CISA é uma referência para os pequenos municípios e que os usuários estão satisfeitos com os servi ços prestados pel o mesmo.

Palavras-chave Satisfação, U suários, Consórcios, Saúde 
Introdução

0 processo de descentralização de ações ede serviços de saúde, ocorrido devido à consolidação do Sistema Ú nico deSaúde (SUS), além detransferir responsabilidades, também redistribuiu re cursos para as esferas estaduais e municipais ${ }^{1}$. Esse processo possibilitou uma melhor qualidade dos serviços oferecidos e sua adaptação às condiç̧̃es locais. Outro fato importante ocorrido foi a ampliação da participação da população e uma elevação da transparência na tomada de decisões². Um dos desafios impostos pelo avanço expressivo da descentralização passou a ser 0 de compatibilizar uma escala adequada de produção de serviços com a proximidade dos problemas locais de saúde da população ${ }^{3}$.

0 planejamento integrado queexpliciteo papel de cada esfera no sistema funcional, definindo competências e responsabilidades de cada ente gestor do SU S por meio de redes regionalizadase resolutivas de saúde, e também a possibilidade de integração intermunicipal voluntária, denominada consorciamento, podem trazer economias de escala, o que possibilita uma maior oferta de serviços de saúde especializados, otimizando, dessa maneira, o uso dos recursos públicos ${ }^{4,5}$

O consórcio é a união de dois ou mais entes da mesma natureza jurídica e constitui um instrumento para solucionar desafios locais, por exemplo, a ampliação e o ordenamento da oferta de serviços; a compra de serviços e a flexibilização da remuneração de profissionais com pagamento de incentivos; ea otimização dos recursos regionais disponíveis, para melhorar 0 acesso e a qualidade dos serviços. Essas ações propiciam benefícios aos usuários e ampliam a resolubilidade da rede de saúde 6 .

A Lei Completar n 82/98 dispõe sobre a criação e implantação de consórcios intermunicipais relacionados à prestação de serviços públicos de interesse comum, nas funções, áreas e setores que especifica eadota outras providências. Considerase consórcio intermunicipal, para efeito da Lei Complementar no 82/98, a sociedadedeM unicípios, integrantes de mesmo aglomerado urbano ou microrregional. Tal se dará previamente autorizada por lei, pela sua Câmara deVereadores, por proposta do Prefeito M unicipal, (com a finalidade de executar serviços público de interesse comum ou obras), adquirir bens, produtos e equipamentos, e, realizar eventos no âmbito da competência municipal.
O consórcio intermunicipal terá direção executiva única, prevista em seus atos constitutivos, e deverá reger-se por estatuto, aprovado por seu conselho diretor previsto na Lei.

No Estado do Paraná, no ano de 1992, a oferta pública de consultas especializadas estava pouco disponível, devido à dificuldade de o governo estadual contratar recursos humanos para atuar nos Centros Regionais de Especialidades. Assim, os consórcios foram estimulados e implantados, visando solucionar o problema. A partir deste momento, os consórcios passaram a assumir, progressivamente, a responsabilidade de organizar e ampliar a assistência médica ambulatorial especializada ${ }^{7}$.

O princípio básico de criação de consórcios desaúde ébastantesimples: quando um município não consegue resolver as necessidades relacionadas à saúde de sua população, um caminho possível para solução é a união com outros municípios com o intuito de garantir a oferta de serviços e um atendimento de boa qualidade ao usuário ${ }^{8}$. Os municípios se associam para resolver problemas específicos de sua região. Assim, cada consórcio adquire peculiaridades e características próprias de sua região?

O M inistério da Saúde considera os consórcios um instrumento eficiente de articulação entre os municípios, sendo a organização de consórcios uma das estratégias importantes para melhorar a qualidade dos serviços oferecidos à população, contribuindo para a consolidação do SU $S^{9}$.

A utilização dos serviços de saúde representa o centro de funcionamento dos sistemas de saúde. 0 conceito de uso compreende todo contato direto - consultas médicas, hospitalizações - ou indireto - realizações de exames preventivos e diagnósticos - com os serviços de saúde. 0 processo de utilização dos serviços de saúde é resultante da interação do comportamento do indivíduo que procura cuidados e do profissional que o conduz dentro do sistema de saúde ${ }^{10}$.

Devido à importância que os consórcios intermunicipais de saúde vêm assumindo na estratégia de descentralização proposto pelo $\mathrm{M} \mathrm{i-}$ nistério da Saúde para um maior acesso da população ao atendimento em nível secundário e otimização dos recursos, a presente pesquisa teve como objetivo conhecer o perfil eavaliar a satisfação dos usuários com os serviços prestados por um consórcio intermunicipal de saúde do noroeste do Paraná. 
Método

Na região noroeste do Paraná, situado no município de Umuarama, o Consórcio Intermunicipal de Saúde (CISA-AM ERIOS/12a Regional de Saúde) é composto por 21 municípios: Alto Piquirí, Altônia, Alto Paraíso, Brasilândia do Sul, Cafezal do Sul, CidadeGaúcha, Cruzeiro do O este, Douradina, Esperança N ova, Francisco Alves, Icaraíma, I porã, I vaté, M aria Helena, M ariluz, Nova Olímpia, Perobal, Pérola, São Jorge do Patrocínio, Tapira e Xambrê.

A distribuição de consultas e procedimentos ofertados pelo CISA para os municípios consorciados é per capita, ou seja, baseado na população de cada município, e o agendamento das mesmas são realizados via online ${ }^{11}$.

Os serviços ofertados pelo CISA são: consultas especializadas, como angiologia, bucomaxilar, cardiologia, cirurgia geral, cirurgia pediátrica, clínica médica, dermatologia, fonoaudiologia, gastroenterologia, gastroenterologia pediátrica, ginecologia eobstetrícia, homeopatia, mastologia, nefrologia, nutrição, oftalmologia, oncologia, ortopedia e traumatologia, otorrinolaringologia, pediatria, pediatria neonatal, psicologia, psiquiatria, urologia, também são disponibilizados exames laboratoriais, raios- $X$, ultrassonografias, mamografias, ressonância magnética, biopsias, entre outros ${ }^{11}$.

Segundo informações do CISA, o mesmo atendeaproximadamentenovecentas pessoas por dia, possibilitando no mínimo trezemil consultas por mês, surgindo a necessidade deampliação de suas instal ações para a melhoria do atendimento, originando o CISA I, CISA II e o CISA III, cada um ofertando serviços de especialidades diferentes ${ }^{11}$.

A presente pesquisa foi realizada na sede do $\mathrm{CISA}$ em Umuarama (PR). Trata-se de um estudo transversal, em que se utilizou um questionário estruturado, constituído de duas partes, a primeira contendo informações socioeconômicas dos usuários e a segunda contendo informações sobre os serviços ofertados pelo CISA.

0 instrumento foi desenvolvido pelos pesquisadores, já que não foram encontrados na literatura instrumentos quantitativos que verificassem o grau de satisfação de usuários dos consórcios intermunicipais eque comparassem com outros sistemas públicos de saúde.

A amostra foi constituída de cem usuários do consórcio intermunicipal de saúde de U muarama.

A pesquisa foi submetida ao comitê de ética em pesquisa com seres humanos na Universidade Paranaense e aprovada sob o n 2066/2006.
Resultados ediscussão

Foram entrevistados cem usuários do serviço saúde, sendo 59 mulheres (59\%) e 41 homens ( $41 \%$ ). Em relação ao gênero dos pacientes, foi encontrado um percentual maior de mulheres atendidas. De acordo com dados de estudos internacionais demorbidade, as mulheres utilizam cerca de $20 \%$ mais os serviços de saúde que os homens ${ }^{12}$. Pinheiro et al. ${ }^{13}$ explicam queébem conhecido o fato que as mulheres fazem maior uso dos serviços de saúde do que os homens, equeessa diferença, em grande parte, érelacionada a questões reprodutivas. Dados encontrados nessa pesquisa são semelhantes à descrita pelos autores ${ }^{12,13}$.

Em relação à idade, $69 \%$ dos usuários entrevistadosestavam na faixa etária acima de 41 anos; isto pode ser explicado pelo aumento de doenças, principalmenteas crônicas, em faixas etárias mais elevadas. A distribuição dos usuários por idade pode ser observada na Tabela 1. A porcentagem de usuários de acordo com o município de origem esta demonstrado na Tabela 2 eo tempo deresidência dos usuários do CISA foi de 14,28 anos em média e pode ser visto Tabela 3.

Em relação ao grau de escolaridade, $27 \%$ dos usuários possuem ensino fundamental ( $1^{\mathrm{a}}$ a $4^{\mathrm{a}}$ séries), $22 \%$ são analfabetos e os outros $51 \%$ se dividem em ensino fundamental ( 5 a a $8^{a}$ ), ensino médio completo eincompleto eterceiro grau incompleto (Figura 1). Esses resultados demonstram um baixo grau de instrução dos usuários atendidos pelo consórcio intermunicipal de saúde. 0 fato de os usuários possuírem um baixo grau deinstrução pode estar diretamenterelacionado à renda baixa; os resultados encontrados

Tabela 1. Distribuição dos usuários do CISA AM ERIOS/12a Regional de Saúde, de acordo com a faixa etária. Paraná, 2006.

\begin{tabular}{lc}
\hline \multicolumn{1}{c}{ I dade } & Porcentagem \% \\
\hline 10 a 20 anos & $4 \%$ \\
21 a 30 anos & $14 \%$ \\
31 a 40 anos & $13 \%$ \\
41 a 50 anos & $28 \%$ \\
51 a 60 anos & $27 \%$ \\
61 a 70 anos & $12 \%$ \\
71 a 80 anos & $2 \%$ \\
Total & $100 \%$ \\
\hline
\end{tabular}

Fonte: Consórcio Intermunicipal deSaúde. 
Tabela 2. Local de origem e porcentagem de usuários entrevistados no CISA - AMERIOS/12ª Regional de Saúde. Paraná, 2006.

\begin{tabular}{lc}
\hline \multicolumn{1}{c}{ M unicípio } & Porcentagem \% \\
\hline Altônia & 15 \\
Tapira & 10 \\
Cafezal do Sul & 8 \\
Douradina & 8 \\
Xambrê & 7 \\
Iporã & 6 \\
Mariluz & 5 \\
Perobal & 5 \\
Icaraíma & 5 \\
Maria Helena & 5 \\
Pérola & 5 \\
Brasilândia do Sul & 4 \\
Alto Paraíso & 2 \\
Alto Piquirí & 2 \\
Cidade Gaúcha & 2 \\
Esperança Nova & 2 \\
Cruzeiro do Oeste & 1 \\
Ivaté & 1 \\
Total & 100
\end{tabular}

Fonte: Consórcio Intermunicipal deSaúde.

Tabela 3. Tempo de residência dos usuários do CISA - AMERIOS/12a Regional de Saúde. Paraná, 2006.

\begin{tabular}{lc}
\hline \multicolumn{1}{c}{ Tempo de residência } & Porcentagem \% \\
\hline 1 a 10 anos & 25 \\
11 a 20 anos & 21 \\
21 a 30 anos & 21 \\
31 a 40 anos & 17 \\
41 a 50 anos & 10 \\
51 a 60 anos & 5 \\
61 a 70 anos & 1 \\
Total & 100 \\
\hline
\end{tabular}

Fonte: Consórcio Intermunicipal deSaúde. na presente pesquisa colaboram com essa hipótese, pois verificou-se que $58 \%$ dos usuários entrevistados recebiam de um a três salários mínimos, sendo estes a maioria aposentados, $42 \%$ dos entrevistados tinham uma renda mensal menor que um salário mínimo, não possuindo emprego fixo. Portanto, verifica-se que essa população depende diretamente dos serviços prestados pelo SUS para resolutividade de seus problemas de saúde, pois não possui renda suficiente para custear 0 atendimento em nível particular ou através de planos de saúde.

Quanto ao número de vezes que os usuários utilizaram os serviços do consórcio, $27 \%$ já utilizaram os serviços de uma a três vezes, $18 \%$, de quatro a seis vezes e $55 \%$, acima de seis vezes.

Em relação à resolutividade dos problemas de saúde, 5\% dos usuários não souberam opinar, $15 \%$ relatam não haver resolutividadee $80 \%$ dos usuários dizem haver sido resolvido o problema que gerou 0 encaminhamento ao serviço. 0 fato da maioria dos usuários relatarem haver resolutividade de seus problemas de saúde levanos a questionar o porquê a mai oria dos usuários utiliza tantas vezes o serviço do consórcio, se realmente os problemas de saúde estão sendo solucionados ou os encaminhamentos repetidos se devem ao fato dos mesmos retornarem para consulta em outra especialidade ou para exames complementares, o que não foi levantado por essa pesquisa.

A Tabela 4 demonstra o grau de satisfação dos usuários do consórcio quanto aos seguintes parâmetros: o tempo em que o município leva para marcar a consulta, o tempo gasto pelo profissional durante a consulta e a opinião sobre os profissionais e sobre as instalações do CISAAMERIOS/12a Regional de Saúde.

Ensino fundamental 1a a $4^{\text {a }}$ - $27 \%$

Ensino fundamental 5a a 8a - 19\%

Ensino médio completo - 17\%

Ensino médio incompleto - 13\%

Terceiro grau incompleto - $2 \%$

Analfabetos - $22 \%$

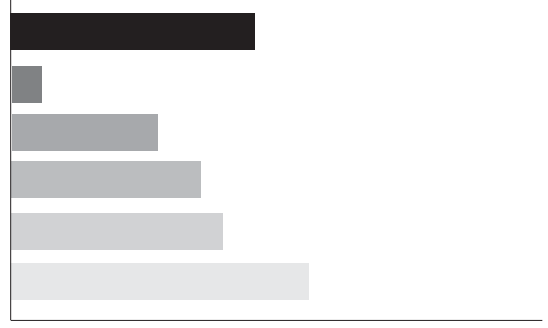

Figura 1. Grau de escolaridade dos usuários do CISA - AM ERIOS/12ª Regional de Saúde. Paraná, 2006. 
Tabela 4. Demonstração do grau de satisfação dos usuários atendidos no CISA - AM ERIOS/12ª Regional de Saúde. Paraná, 2006.

\begin{tabular}{lrrrrrr}
\hline & Ótimo & Bom & Razoável & Ruim & Péssimo & Total \\
\hline $\begin{array}{l}\text { Como você classifica o tempo que o } \\
\text { município levou para marcar a consulta }\end{array}$ & $11 \%$ & $57 \%$ & $13 \%$ & $16 \%$ & $3 \%$ & $100 \%$ \\
Tempo gasto durante a consulta & $9 \%$ & $43 \%$ & $32 \%$ & $14 \%$ & $2 \%$ & $100 \%$ \\
Opinião sobre o profissional & $25 \%$ & $68 \%$ & $6 \%$ & $1 \%$ & $0 \%$ & $100 \%$ \\
Como você avalia as instalações do CISA & $56 \%$ & $41 \%$ & $2 \%$ & $1 \%$ & $0 \%$ & $100 \%$
\end{tabular}

Fonte: Consórcio Intermunicipal deSaúde.

O tempo que o município leva para marcar a consulta no consórcio depen de da especialidade; em média, 44\% dos usuários responderam levar menos de um mês e $46 \%$ relataram que para ser marcada uma consulta o tempo de espera é de um a três meses. Esse intervalo de espera para a marcação da consulta foi considerado bom pelos usuários entrevistados.

Em relação ao tempo gasto durante a consulta, $16 \%$ dos usuários relatam queéinsuficiente para que um bom diagnóstico seja realizado, argumentando "que muitos médicos nem esperam o pacientes relatar seu problema e já prescrevem o medicamento". Para $43 \%$ dos pacientes, o tempo de consulta foi considerado bom e $32 \%$ relataram o tempo como sendo razoável. Os autores enfatizam que um maior tempo de consulta está associado a uma melhor qualidade no atendimento: com uma melhor anamnese, uma melhor explicação do problema e dos procedimentos dos diagnósticos e terapêuticos, assim como a verificação do médico sobre a compreensão do paciente e participação do paciente na consulta ${ }^{14}$.

Em relação ao profissional médico, $68 \%$ dos pacientes os consideram bons profissionais. Segundo os autores, a maioria das queixas que os pacientes fazem éa respeito de problemas comunicacionais com o médico enão a sua competênciaclínica ${ }^{14}$.

Quanto os usuários foram indagados a respeito das instalações físicas do CISA, como por exemplo, recepção, refeitório e consultórios, foram considerados ótimo e bom por $97 \%$ dos usuários.

\section{Conclusão}

0 atendimento de nível secundário preconizado pelo Sistema Único deSaúdeé de responsabilidadedos estados; porém, o município estámais perto dos usuários, então os mesmos procuram as secretarias municipais de saúde para a resolutividade de seus problemas de saúde, pois não conhecem a responsabilidade de cada esfera de governo, tendo assim o município que arcar com esse ônus. Como as verbas repassadas para 0 atendimento são escassas, os consórcios tornaram-se uma maneira viável para atender os cidadãos que necessitam de um especialista, racionalizando e otimizando os escassos recursos repassados para 0 atendimento de especialidades dos usuários.

Concluiu-se com essa pesquisa que o CISA deU muarama está nesse contexto, devido ao perfil dos usuários: os mesmos não podem pagar um plano de saúde, somente tem acesso a esse serviço através do Sistema Ú nico de Saúde(SUS) e estão satisfeitos com o atendimento prestado pelo mesmo.

\section{Colaboradores}

M Greco participou do levantamento dos dados decampo ena concepção teórica. EV M uller participou da análise dos dados de campo, da concepção teórica, elaboração, revisão e redação final do texto. 


\section{Referências}

1. Brasil. M inistério da Saúde Proposta de Regionalização da Assistência à Saúde. 2a versão preliminar para discussão interna do M S e exposição à CIT em 20/7/ 2000. Braślia: M inistério da Saúde; 2000.

2. Brasil. M inistério da Saúde. Norma Operacional Básica da Assistência à Saúde do SUS (N OAS-SUS 01/ 01). Portaria MS/GM, n. 95, de 26 de janeiro de 2001 e regulamentações. Brasília: M inistério da Saúde; 2001.

3. Teixeira L, Macdowel MC, Bugarin M. Incentivos em consórcios intermunicipais de saúde: uma abordagem de teoria dos contratos. Brasília: UNB; 2002. [Série textos para discussão $n^{\circ} 246$ ]

4. Bertone AA. Consórcios intermunicipais de saúde - uma estratégia rumo à regionalização. Revista Divulgação em Saúde para Debate 1996; 16:36-39.

5. Vaz JC, Bava EC. Consórcios Intermunicipais. [site da Internet] [acessado 2005 out 26]. Disponível em: http://www.federativo.bndes.bov.br

6. Mendes EV. Os grandes dilemas do SUS. Salvador: Casa da Qualidade Editora; 2001.

7. Nicoletto SC, Cordoni L, Costa RN. Consórcios intermunicipais de saúde: 0 caso do Paraná, Brasil. Cad Saude Publica [periódico na Internet] 2005 [acessado 2005 out 26];21(1). Disponível em: http:/ /www.scielo.br/pdf/csp/v21n1/04.pdf

8. Coelho JRGP, Veloso C. Consórcios Intermunicipais de Saúde em M inas Gerais. Divulgação em Saúde para Debate 1997; 17:36-38.

9. Brasil. Ministério da Saúde. 0 Consórcio e a Gestão M unicipal em Saúde. Brasília: M inistério da Saúde; 1997.

10. Travassos C, Martins M. U ma revisão sobre os conceitos de acesso e utilização de serviços de saúde. Cad Saude Publica 2004; 20(2):S190-S198.

11. Castanha A. Jornal do CISA 2006; 2(2):1-12.

12. Pereira MG. Epidemiologia: teoria e prática. Rio de Janeiro: Guanabara Koogan; 2005.

13. Pinheiro RS, Viacava F, Travassos C, Britto AS. Gênero, morbidade, acesso e utilização de serviços de saúde no Brasil. Cien Saude Colet 2002; 7(4):687-707.

14. Caprara A, Rodrigues J. A relação assimétrica médico-paciente: repensando o vínculo terapêutico. Cien Saude Colet 2004; 9(1):139-146.

Artigo apresentado em 25/01/2007

Aprovado em 25/10/2007

Versão final apresentada em 04/12/2007 